\title{
Stellar velocity dispersions in AGN - II: Methods
}

\author{
Natalia Vale Asari ${ }^{1}$, Aurea Garcia-Rissmann ${ }^{1}$, Luis Rodolfo Vega \\ Neme $^{1}$ and Roberto Cid Fernandes ${ }^{1}$ \\ ${ }^{1}$ Universidade Federal de Santa Catarina, Florianópolis, SC, Brazil
}

\begin{abstract}
Stellar velocity dispersions in AGN are useful indicators of the black-hole mass (through the $M_{\bullet}-\sigma_{\star}$ relation) and nuclear stellar populations (through the $M / L$ ratio). We have collected near-infrared spectra of $\sim 40$ Seyferts in the CaII triplet range in order to measure $\sigma_{\star}$ and investigate the connection between stellar populations and AGN properties. In this poster we present a comparison between two methods to measure velocity dispersions: (1) direct fitting (DFM) and (2) cross-correlation (CCM). (1) In DFM the spectra in the CaII triplet range is modeled as a combination of a broadened and shifted stellar spectrum (observed through the same instrumental setup) and a featureless continuum. This method has the advantage of making it easy to mask out noisy regions and sky residuals. (2) CCM converts the width of the star-galaxy cross-correlation function onto a velocity dispersion. We find that these methods yield velocity dispersions consistent to within $20 \mathrm{~km} \mathrm{~s}^{-1}$ on-average. Our results are consistent to within $20 \mathrm{~km} \mathrm{~s}^{-1}$ with those objects available in the literature.
\end{abstract}

\section{Introduction}

Stellar velocity dispersions $\left(\sigma_{\star}\right)$ are a measure of the stellar kinematics in a galaxy. Due to its location in a relatively clean spectral region, the CaII absorption triplet (CaT, $8498,8542,8662 \AA)$ is a useful tracer of $\sigma_{\star}$. A reliable method to measure stellar velocity dispersions in AGN is required, so we study two of them: DFM and CCM.

For DFM we developed a program based on our Stellar Population Synthesis algorithm. DFM is basically a Gaussian filter that broadens and smoothes template stellar spectra and obtains a model spectrum, whose parameters $\left(\sigma_{\star}, v_{\star}\right.$, etc. $)$ are adjusted until an optimal match is achieved.

CCM has been extensively used mainly because it demands less computer processing. We used the task fxcor in IRAF, which finds the cross-correlation function between the galaxy and a star template in Fourier space. The peak of the cross-correlation function is then modelled by a Gaussian, which yields its $\mathrm{FWHM}_{C C F}$. We find the relation $\mathrm{FWHM}_{C C F^{-}} \sigma_{\star}$ by an empirical calibration with the template stars.

\section{Direct Fitting Method and Conclusions}

The Direct Fitting Method (DFM) fits a model, $M_{\lambda}$, to the observed spectrum:

$$
M_{\lambda}=M_{\lambda_{0}}\left[\sum_{j=1}^{N_{\star}} x_{j} T_{j, \lambda} r_{\lambda}\right] \otimes G\left(v_{\star}, \sigma_{\star}\right)
$$

where (a) $M_{\lambda_{0}}$ is the total synthetic flux at $\lambda_{0}$; (b) $T_{j, \lambda}$ is the spectrum of the $j^{\text {th }}$ ( $j=$ $\left.1, \ldots, N_{\star}\right)$ template normalized at $\lambda_{0}$, and $x_{j}$ is its fractional contribution to $M_{\lambda_{0}}$. For convenience, we include a featureless continuum $C_{\lambda}$ in the base of templates. $C_{\lambda}$ allows the model to match the dilution of the galaxy spectrum and is constituted by a weighted sum 

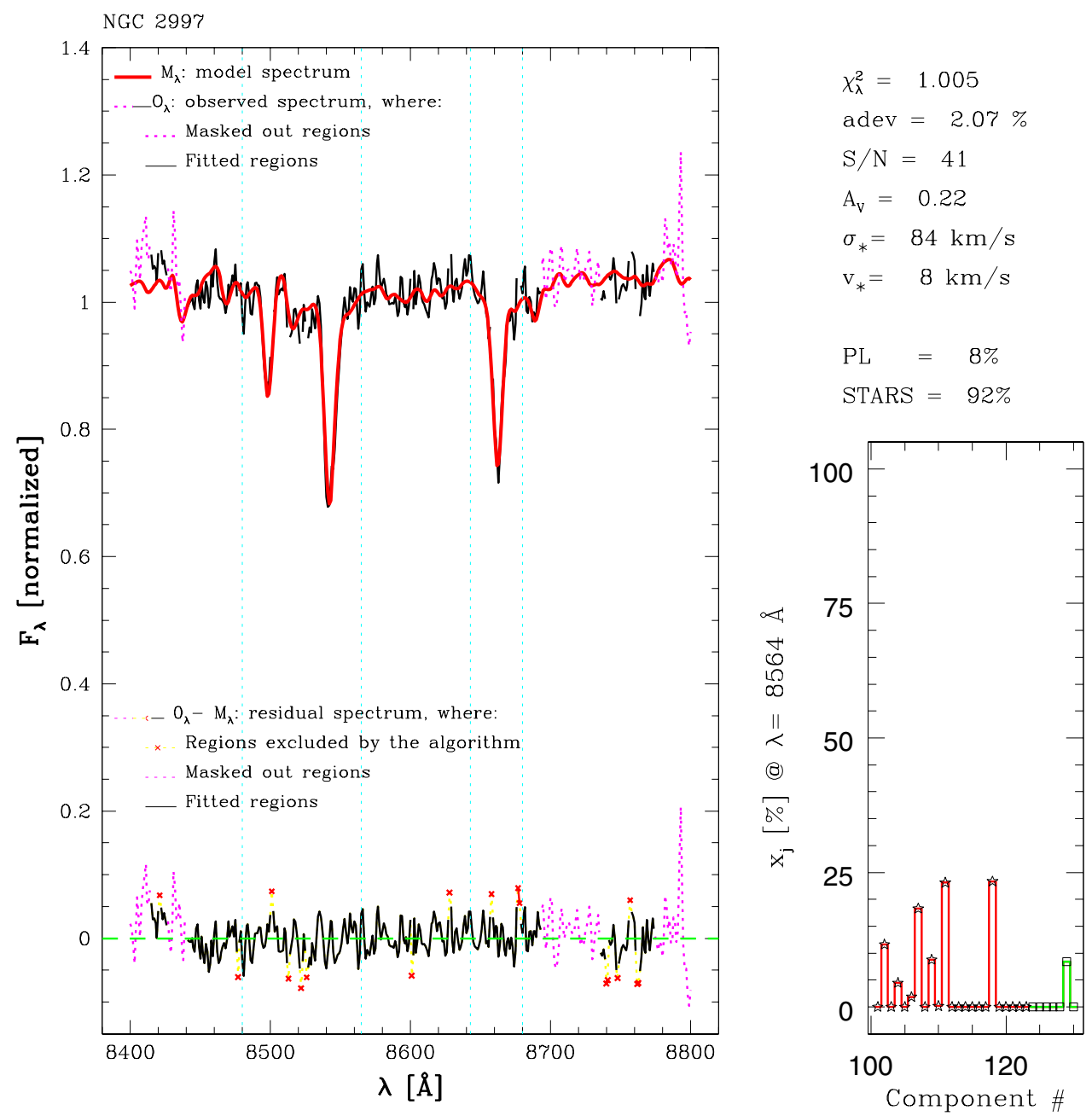

Figure 1. DFM Results for NGC 2997. The direct fitting and the cross-correlation methods yield velocity dispersions consistent to within $20 \mathrm{~km} \mathrm{~s}^{-1}$. Our results are consistent to within $20 \mathrm{~km} \mathrm{~s}^{-1}$ with those objects available in the literature. As future steps, we will have more rigorous estimates of the uncertainties in $\sigma_{\star}$; use $\sigma_{\star}$ to estimate the stellar mass $M_{\star}$ and $M / L$; use $\sigma_{\star}$ to estimate $M_{\bullet}$; and investigate relations among $M / L$, stellar populations and $M_{\bullet}$.

of seven power-laws; (c) $r_{\lambda}=10^{-0.4\left(A_{\lambda}-A_{\lambda_{0}}\right)}$ accounts for the reddening; (d) $G\left(v_{\star}, \sigma_{\star}\right)$ is a Gaussian line-of-sight velocity distribution, centred at $v_{\star}$ and broadened by $\sigma_{\star}$.

We first perform DFM in the CaT region (8480-8565, 8643-8680 $\AA$ ). After analysing these preliminary fits, we construct individual masks for each galaxy, avoiding unwanted spectral features, such as emission lines, bad pixels and sky residuals. This is one of the main advantages of DFM, as it makes it easy to construct masks and compare the model and the observed spectra.

\section{Acknowledgements}

This work is supported by $\mathrm{CNPq}$ and UFSC (PIBIQ/CNPq - BIP/UFSC). 